\title{
Predictors of no-Reflow in Patients with ST-Segment Elevation Acute Myocardial Infarction after Primary Percutaneous Coronary Intervention
}

\author{
Mohammed Ismail Ali ${ }^{1}$, Wala Farid Abd El-Aziz ${ }^{2}$, Adel Imam Abd El-Magid ${ }^{3}$, \\ Neveen Ibrahim Samy ${ }^{2}$ \\ ${ }^{1}$ Cardiology Department, 6 October Hospital, General Health Insurance, Giza, Egypt \\ ${ }^{2}$ Cardiology Department, Menoufia University, Menoufia, Egypt \\ ${ }^{3}$ Cardiology Department, National Heart Institute, Giza, Egypt
}

Email address:

Mohammed.Ismail.Anwar@gmail.com (M. I. Ali)

To cite this article:

Mohammed Ismail Ali, Wala Farid Abd El-Aziz, Adel Imam Abd El-Magid, Neveen Ibrahim Samy. Predictors of no-Reflow in Patients with ST-Segment Elevation Acute Myocardial Infarction after Primary Percutaneous Coronary Intervention. Cardiology and Cardiovascular Research. Vol. 2, No. 3, 2018, pp. 68-74. doi: 10.11648/j.ccr.20180203.15

Received: August 28, 2018; Accepted: October 15, 2018; Published: November 5, 2018

\begin{abstract}
Background: Primary percutaneous coronary intervention (PCI) is the preferred method of ST-segment elevation acute myocardial infarction (STEMI) management but no-reflow phenomenon is one of its major complications that affects patient's outcome. Objective: Identification of possible clinical, angiographic and procedural predictors for no-reflow in patients with AMI after primary percutaneous coronary intervention (PCI). Methods: A total of hundred patients with AMI who had been treated by primary PCI at the National heart institute (NHI) and cardiology department, Menoufia University hospital were enrolled in this study, according to thrombolysis in myocardial infarction (TIMI) flow grade, patients were divided into a reflow group (TIMI 3 ) and a no-reflow group (TIMI $\leq 2$ ). The clinical, angiographic and procedural data were compared between both groups. Discussion: Sixteen patients (16\%) developed no-reflow phenomenon after primary PCI. Statistical analysis showed that time from onset to reperfusion, low initial TIMI flow grade, high thrombus burden, long lesion length and large reference luminal diameter were correlated with no-reflow $(\mathrm{P}<0.05$ for all) and were considered to be independent predictors of no-reflow. Conclusion: The occurrence of no-reflow after primary PCI for acute myocardial infarction can be predicted by certain clinical, angiographic and procedural features.
\end{abstract}

Keywords: Acute Myocardial Infarction, Primary Percutaneous Coronary Intervention, No-Reflow, Coronary Thrombosis

\section{Introduction}

The treatment of ST-segment elevation myocardial infarction (STEMI) is directed toward rapid and proper restoration of normal blood flow in the infarct related artery (IRA) to decrease ischemic damage to the myocardium as early as possible [1]. Percutaneous coronary intervention (PCI) is the most suitable method to achieve this goal in addition to limitation of infarction size and improvement of prognosis in patients with acute myocardial infarction (AMI) [2]. Patency of the infarct-related artery does not always mean restoration of normal coronary blood flow as in certain group of patients epicardial coronary artery reperfusion is achieved but without myocardial reperfusion after primary $\mathrm{PCI}$, this condition is known as no-reflow [3]. Patients who develop this phenomenon are subjected to more myocardial damage and higher risk of cardiac morbidity and mortality [4].

\section{Patients and Methods}

\subsection{Patients Populations}

Total of hundred AMI patients who underwent primary PCI between January 2016 and June 2016 at the National heart institute (NHI) and cardiology department, Menoufia University hospital were enrolled in the study. AMI was defined as typical chest pain with either ST-segment elevation of $>1 \mathrm{~mm}$ in 2 
consecutive leads or the new onset of left bundle-branch block with 2-fold elevation of creatine kinase (CK) and creatine kinase-myocardial band (CK-MB) fraction [5].

\subsection{Inclusion Criteria}

1. Patients underwent primary PCI within 12 hours after onset of symptoms.

2. Patients with ongoing chest pain for more than 12 hours who underwent primary PCI within 24 hours after onset of symptoms.

\subsection{Exclusion Criteria}

1. Patients with coronary artery spasm or $<50 \%$ diameter stenosis of the culprit lesion.

2. Patients who required emergency surgical revascularization for significant left main coronary artery disease or multi-vessel disease.

3. Patients with saphenous vein grafts or left internal mammary artery lesions.

4. Patients did not achieved coronary artery patency.

5. Presence of mechanical complications such as dissection or angiographically evident distal embolization after completion of the procedure.

\subsection{Methods}

All the study population was subjected to the following:

Full history taking including age, gender, risk factors for coronary artery disease such as diabetes mellitus, hypertension, smoking, dyslipidemia and family history of premature coronary artery disease.

Physical examination to detect signs of heart failure and mechanical complications.

12-Lead ECG documented either ST-segment elevation of $>1 \mathrm{~mm}$ in 2 consecutive leads or new onset left bundlebranch block.

Cardiac biomarkers including cardiac troponins, $\mathrm{CK}$ and $\mathrm{CK}-\mathrm{MB}$ and serum creatinine.
All patients received oral aspirin (300 mg) and clopidogrel $(600 \mathrm{mg})$ as well as intravenous $8.000-10.000$ IU of unfractionated heparin.

Standard coronary angiograms was carried out through the femoral approach with assessment of the following angiographic data:

Identification of the IRA, angiographic features of the culprit lesion, TIMI flow grade before and after primary PCI, target lesion length, reference luminal diameter, type of total occlusion if present (tapered or cut-off lesion), type of subtotal occlusion if present (eccentric or concentric lesion), lesion location (proximal, mid or distal), thrombus burden degree (mild, moderate or high) was scored according to the thrombus scoring system proposed by the TIMI group, it was classified as mild if the TIMI thrombus grade was class 0 or 1 , moderate if the TIMI thrombus grade was class 2 or 3 , and high if the TIMI thrombus grade was more than class 3 [6].

All patients were treated by bare metal stents restricted to the culprit lesion according to the insurance policy.

Platelets glycoprotein IIb/IIIa inhibitors were used on downstream basis according to the thrombus burden degree.

The whole study group was classified into two groups according to the post-PCI TIMI flow: Group I with angiographically documented TIMI flow 3 (reflow) and Group II with angiographically documented TIMI flow $\leq 2$ (no-reflow).

The patient was considered to exhibit a no-reflow phenomenon if blood flow in the IRA was TIMI $\leq 2$ flow despite successful dilatation and absence of mechanical complications such as dissection or angiographically evident distal embolization after completion of the procedure.

\section{Results}

As shown in table 1 and 2 and figure 1 the two study groups were compared regarding demographic and clinical data with statistically significant longer reperfusion time which is defined as time from the onset of symptoms to the onset of reperfusion in the No-reflow group.

Table 1. The demographic and clinical categorical data of both study groups.

\begin{tabular}{|c|c|c|c|c|c|c|c|}
\hline \multirow{3}{*}{ Variables } & & \multicolumn{4}{|c|}{ Post-PCI TIMI flow } & \multirow{2}{*}{\multicolumn{2}{|c|}{ Chi-square }} \\
\hline & & \multicolumn{2}{|c|}{ Reflow } & \multicolumn{2}{|c|}{ No-reflow } & & \\
\hline & & $\mathbf{N}$ & $\%$ & $\mathbf{N}$ & $\%$ & $\mathbf{X}^{2}$ & P-value \\
\hline \multicolumn{2}{|l|}{ Male } & 57 & $67.9 \%$ & 12 & $75 \%$ & 0.331 & 0.565 \\
\hline \multicolumn{2}{|c|}{ Current smoking } & 51 & $60.7 \%$ & 9 & $56.3 \%$ & 0.111 & 0.739 \\
\hline \multicolumn{2}{|c|}{$\mathrm{DM}$} & 31 & $36.9 \%$ & 6 & $37.5 \%$ & 0.002 & 0.964 \\
\hline \multicolumn{2}{|c|}{ HTN } & 44 & $52.4 \%$ & 9 & $56.3 \%$ & 0.081 & 0.776 \\
\hline \multicolumn{2}{|c|}{ Dyslipidemia } & 41 & $48.8 \%$ & 7 & $43.8 \%$ & 0.138 & 0.710 \\
\hline \multicolumn{2}{|c|}{ Prior PCI } & 30 & $35.7 \%$ & 5 & $31.3 \%$ & 0.119 & 0.730 \\
\hline \multicolumn{2}{|l|}{ Prior MI } & 24 & $28.6 \%$ & 5 & $31.3 \%$ & 0.046 & 0.830 \\
\hline \multirow{4}{*}{ Killip class } & Killip I & 57 & $67.9 \%$ & 7 & $43.8 \%$ & \multirow{4}{*}{3.462} & \multirow{4}{*}{0.326} \\
\hline & Killip II & 20 & $23.8 \%$ & 6 & $37.5 \%$ & & \\
\hline & Killip III & 5 & $6.0 \%$ & 2 & $12.5 \%$ & & \\
\hline & Killip IV & 2 & $2.4 \%$ & 1 & $6.3 \%$ & & \\
\hline
\end{tabular}

DM: Diabetes Mellitus

HTN: Hypertension

PCI: Percutaneous coronary intervention

MI: Myocardial infarction 
Table 2. The demographic and clinical continuous data of both study groups.

\begin{tabular}{|c|c|c|c|c|c|c|c|c|}
\hline \multirow{3}{*}{ Variables } & \multicolumn{6}{|c|}{ Post-PCI TIMI flow } & \multirow{2}{*}{\multicolumn{2}{|c|}{ T-test }} \\
\hline & \multicolumn{3}{|c|}{ Reflow } & \multicolumn{3}{|c|}{ No-reflow } & & \\
\hline & Mean & \pm & SD & Mean & \pm & SD & $\mathbf{t}$ & P-value \\
\hline Age (Y) & 53.310 & \pm & 10.236 & 57.313 & \pm & 9.443 & -1.450 & 0.150 \\
\hline HR (bpm) & 100.905 & \pm & 20.150 & 99.875 & \pm & 21.731 & 0.185 & 0.854 \\
\hline SBP (mmHg) & 112.381 & \pm & 27.369 & 116.875 & \pm & 31.563 & -0.587 & 0.558 \\
\hline $\mathrm{DBP}(\mathrm{mmHg})$ & 69.226 & \pm & 15.878 & 72.813 & \pm & 18.883 & -0.803 & 0.424 \\
\hline Peak CK-MB (IU/ml) & 51.345 & \pm & 17.081 & 46.625 & \pm & 18.807 & 0.997 & 0.321 \\
\hline Reperfusion time (hours) & 5.857 & \pm & 3.038 & 8.000 & \pm & 4.082 & -2.439 & $0.017 *$ \\
\hline
\end{tabular}

HR: heart rate

Bpm: beat per minute

CK-MB: Creatine kinase myocardial band

SBP: systolic blood pressure

DBP: diastolic blood pressure

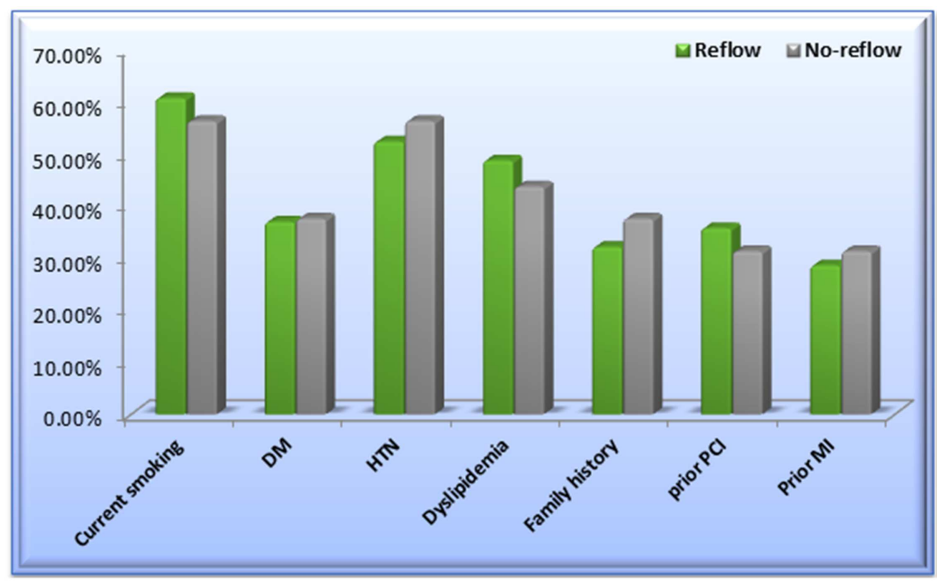

Figure 1. The demographic and clinical data of both study groups.

As shown in table 3 and figure 2 both groups were compared regarding the angiographic data with statistically significant higher thrombus burden degree and lower initial TIMI flow grade in the No-reflow group.

Table 3. The angiographic categorical data of both study groups.

\begin{tabular}{|c|c|c|c|c|c|c|c|}
\hline \multirow{3}{*}{ Variables } & & \multicolumn{4}{|c|}{ Post-PCI TIMI flow } & \multirow{2}{*}{\multicolumn{2}{|c|}{ Chi-square }} \\
\hline & & \multicolumn{2}{|c|}{ Reflow } & \multicolumn{2}{|c|}{ No-reflow } & & \\
\hline & & $\mathbf{N}$ & $\%$ & $\mathbf{N}$ & $\%$ & $\mathbf{X}^{2}$ & P-value \\
\hline \multirow{3}{*}{ Infarction location } & anterior & 55 & $65.5 \%$ & 10 & $62.5 \%$ & \multirow{3}{*}{0.231} & \multirow{3}{*}{0.891} \\
\hline & inferior & 26 & $31.0 \%$ & 5 & $31.3 \%$ & & \\
\hline & other & 3 & $3.6 \%$ & 1 & $6.3 \%$ & & \\
\hline \multirow{3}{*}{ IRA } & LAD & 55 & $65.5 \%$ & 10 & $62.5 \%$ & \multirow{3}{*}{0.209} & \multirow{3}{*}{0.794} \\
\hline & LCX & 10 & $11.9 \%$ & 2 & $12.5 \%$ & & \\
\hline & $\mathrm{RCA}$ & 19 & $22.6 \%$ & 4 & $25.0 \%$ & & \\
\hline \multirow{3}{*}{ Target lesion location } & Proximal & 31 & $36.9 \%$ & 7 & $43.8 \%$ & \multirow{3}{*}{0.264} & \multirow{3}{*}{0.608} \\
\hline & mid & 45 & $53.6 \%$ & 7 & $43.8 \%$ & & \\
\hline & distal & 8 & $9.5 \%$ & 2 & $12.5 \%$ & & \\
\hline \multirow{3}{*}{ Thrombus burden } & High & 33 & $39.3 \%$ & 9 & $56.3 \%$ & \multirow{3}{*}{4.984} & \multirow{3}{*}{$0.047 *$} \\
\hline & Moderate & 21 & $25.0 \%$ & 4 & $25.0 \%$ & & \\
\hline & Low & 30 & $35.7 \%$ & 3 & $18.8 \%$ & & \\
\hline \multirow{2}{*}{$\begin{array}{l}\text { Total } \\
\text { occlusion morphology }\end{array}$} & Cut-off & 33 & $39.3 \%$ & 8 & $50.0 \%$ & \multirow{2}{*}{0.529} & \multirow{2}{*}{0.487} \\
\hline & Tapered & 27 & $32.1 \%$ & 4 & $25.0 \%$ & & \\
\hline \multirow{2}{*}{$\begin{array}{l}\text { Subtotal occlusion } \\
\text { morphology }\end{array}$} & Concentric & 11 & $13.1 \%$ & 2 & $12.5 \%$ & \multirow{2}{*}{0.097} & \multirow{2}{*}{0.755} \\
\hline & Eccentric & 13 & $15.5 \%$ & 2 & $12.5 \%$ & & \\
\hline \multirow{2}{*}{ Initial TIMI flow } & $\mathrm{T} 0 / 1$ & 53 & $63.1 \%$ & 13 & $81.2 \%$ & \multirow{2}{*}{5.342} & \multirow{2}{*}{$0.043 *$} \\
\hline & $\mathrm{T} 2 / 3$ & 31 & $36.9 \%$ & 3 & $18.8 \%$ & & \\
\hline
\end{tabular}

IRA: Infarct related artery

LAD: Left anterior descending

LCX: Left circumflex

RCA: Right coronary artery

TIMI: Thrombolysis in myocardial infarction 


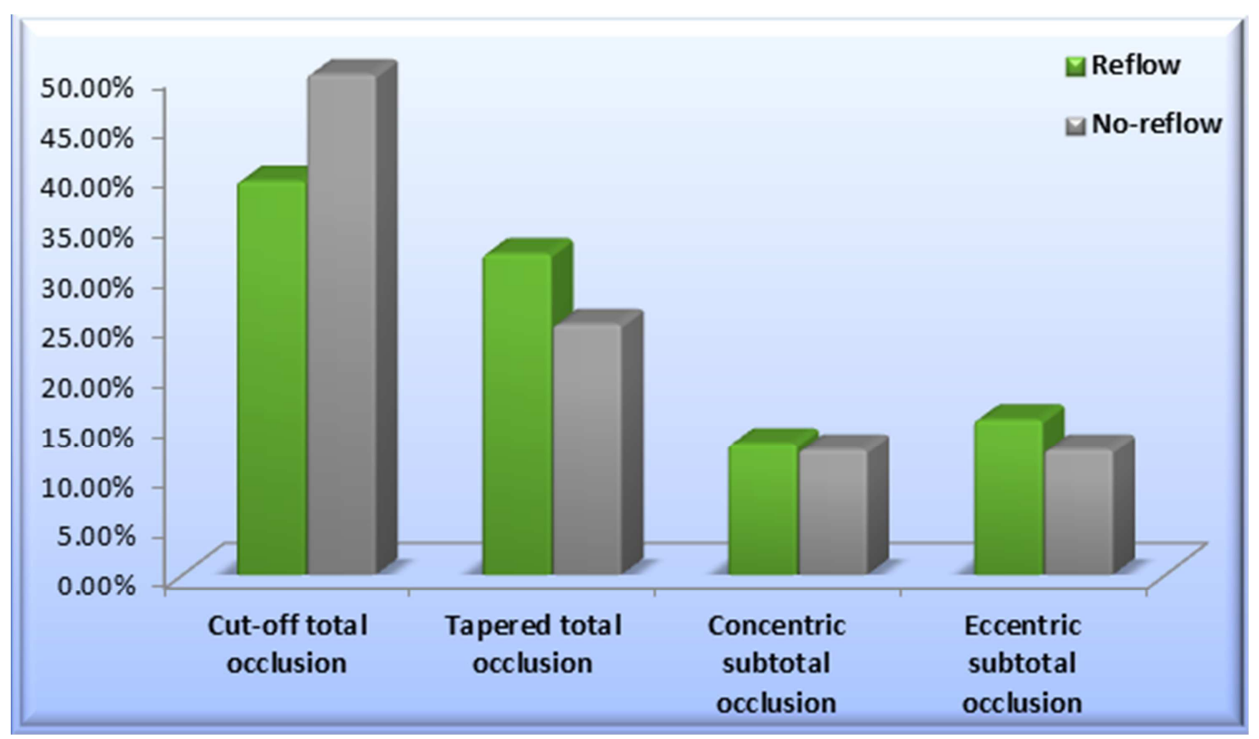

Figure 2. The lesion morphology of both study groups.

As shown in tables 4 and 5 and figure 3 both groups were compared regarding the procedural data with statistically significant larger reference luminal diameter and longer lesion length in the No-reflow group.

Table 4. The procedural categorical data of both study groups.

\begin{tabular}{|c|c|c|c|c|c|c|}
\hline \multirow{3}{*}{ Variables } & \multicolumn{4}{|c|}{ Post-PCI TIMI flow } & \multirow{2}{*}{\multicolumn{2}{|c|}{ Chi-square }} \\
\hline & \multicolumn{2}{|c|}{ Reflow } & \multicolumn{2}{|c|}{ No-reflow } & & \\
\hline & $\mathbf{N}$ & $\%$ & $\mathbf{N}$ & $\%$ & $\mathrm{X}^{2}$ & P-value \\
\hline Stenting after predilatation & 34 & $40.5 \%$ & 7 & $43.8 \%$ & 0.059 & 0.808 \\
\hline Direct stenting & 50 & $59.5 \%$ & 9 & $56.3 \%$ & 0.059 & 0.808 \\
\hline Single stent & 67 & $79.8 \%$ & 12 & $75.0 \%$ & 0.177 & 0.674 \\
\hline Multiple stents & 17 & $20.2 \%$ & 4 & $25.0 \%$ & 0.177 & 0.674 \\
\hline Aspiration device use & 49 & $58.3 \%$ & 10 & $62.5 \%$ & 0.097 & 0.755 \\
\hline $\begin{array}{l}\text { GP IIb/IIIa inhibitors use } \\
\text { GP: Glycoprotein }\end{array}$ & 55 & $65.5 \%$ & 11 & $68.8 \%$ & 0.065 & 0.799 \\
\hline
\end{tabular}

Table 5. The procedural continuous data of both study groups.

\begin{tabular}{|c|c|c|c|c|c|c|c|c|}
\hline \multirow[t]{3}{*}{ Variables } & \multicolumn{6}{|c|}{ Post-PCI TIMI flow } & \multirow{2}{*}{\multicolumn{2}{|c|}{ T-test }} \\
\hline & \multicolumn{3}{|c|}{ Reflow } & \multicolumn{3}{|c|}{ No-reflow } & & \\
\hline & Mean & \pm & SD & Mean & \pm & SD & $t$ & P-value \\
\hline Reference diameter mm & 3.247 & \pm & 0.361 & 3.500 & \pm & 0.365 & -2.565 & $0.012 *$ \\
\hline Target lesion length mm & 22.583 & \pm & 5.811 & 25.625 & \pm & 4.646 & -1.974 & $0.05 *$ \\
\hline
\end{tabular}

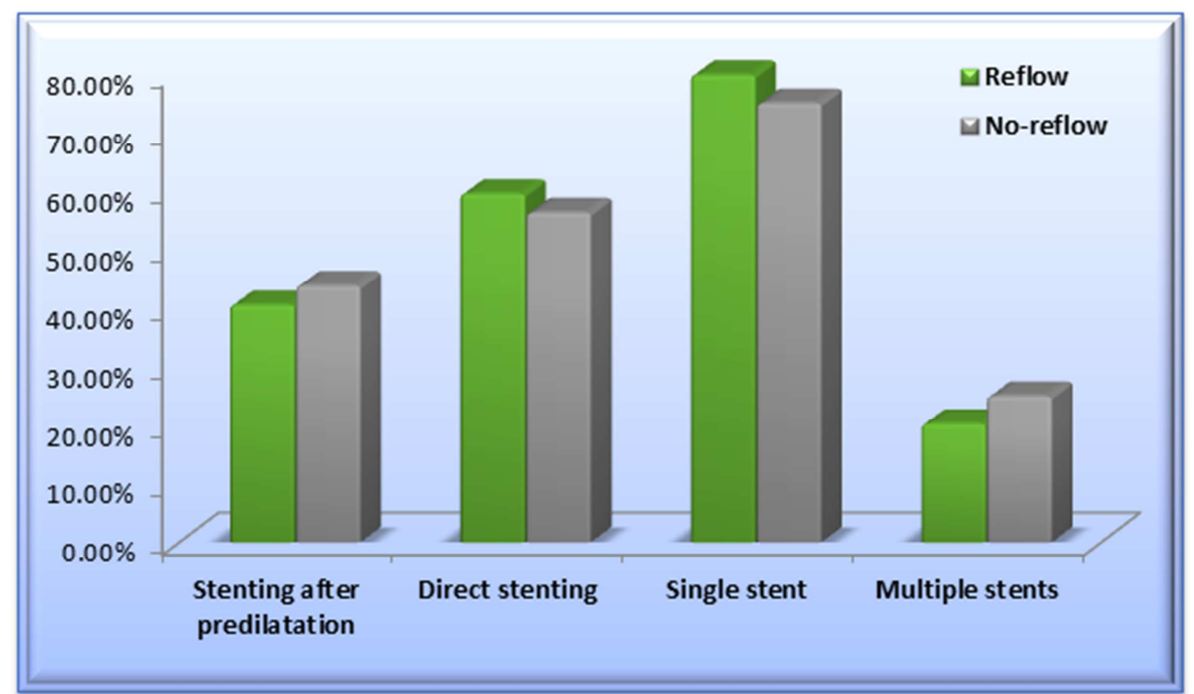

Figure 3. The procedural data of both study groups. 


\section{Discussion}

This study included one hundred patients who underwent primary percutaneous coronary intervention at the National heart institute (NHI) and cardiology department, Menoufia University hospital within the period between January 2016 and June 2016.

All patients were treated by bare metal stents (BMS). In the Norwegian Coronary Stent (NORSTENT) trial, 9013 patients underwent PCI (26\% with STEMI) were randomized to DES or BMS with no differences in the incidence of the primary endpoint (Death from any cause or non-fatal MI) after a median follow-up of 5-years [7].

Stenting was restricted to the culprit lesion only and timing of non-IRA revascularization (before or after hospital discharge) was determined by the treating physician. Recent data recommend that revascularization of non-IRA lesions should be considered in STEMI patients with multi-vessel disease before hospital discharge. The ideal timing of revascularization (immediate or staged) has not been properly investigated [8].

Platelets glycoprotein IIb/IIIa inhibitors were used on downstream basis according to thrombus burden degree. The routine upstream use of glycoprotein (GP) IIb/IIIa inhibitors before primary PCI was not associated with clinical benefit and it was associated with higher bleeding risk compared with downstream use in the catheterization laboratory [9].

Deferred stenting strategy was not employed according to the physician discretion.

This strategy was investigated as a method to reduce micro-vascular obstruction (MVO) and to preserve microcirculatory function but it was associated with higher rate of target vessel revascularization. So, its routine use is not recommended [10].

The whole study group was classified into two groups according to the post-PCI TIMI flow grade: Group I included 84 patients with angiographically documented TIMI flow grade 3 (reflow) and Group II included another 16 patients with angiographically documented TIMI flow grade $\leq 2$ (noreflow).

Coronary angiography is the simplest method to diagnose no-reflow in the catheterization laboratory. TIMI flow grade and myocardial blush grade (MBG) are the most commonly used methods to define angiographic no-reflow. TIMI flow grade refers to the intensity and extent of visualization of IRA and the speed of flow of dye. TIMI flow is graded 0-3. MBG refers to the intensity of radio-opacity of the myocardial tissue and the speed with which the enhancement clears. MBG is also graded as $0-3$ [16].

Myocardial blush grade was not used as most angiogram films were not acquired long enough to estimate the MBG and the TIMI flow grade classification system was used only to assess epicardial coronary blood flow. This was concordant to many studies $[1,5,11-14]$ but discordant to Chen who used TIMI flow grade system and MBG to define no-reflow [15].
In the present study, no-reflow occurs in $16.0 \%$ of study population. This incidence is lower than that reported by Watanabe (26.0\%), Chen (25.3\%), Mazhar (25.0\%), Morishima (25.0\%) and Kirma (24.3\%), close to that reported by Zhou (17.3\%) and higher than that reported by Tanaka (13.0\%) [1, 5, 11-15,].

Many experimental and clinical data have shown that the incidence of no-reflow ranging from $5 \%$ to $25 \%$ according to the methods of assessment and the population under study [17].

The two study groups were compared regarding demographic and clinical data with statistically significant longer reperfusion time in the No-reflow group ( $8.0 \pm 4.0$ vs. $5.8 \pm 3.0$ hours for no-reflow and reflow, respectively).

Significant longer reperfusion time in patients with noreflow was concordant to many studies [1, 5, 14] but discordant to others [11-13, 15].

Delayed presentation is a potentially preventable factor and associated with more ischemic injury that results in swelling of myocardial cells, edema of capillary bed and neutrophil plugging [18].

In the early stages of AMI, the thrombus contains more thrombocytes and is more vulnerable to lyses but with longer reperfusion time, the rigidity of the thrombus increases and it tends to fragment with balloon dilatation which can lead to distal coronary embolization during primary PCI [19].

The rate of no-reflow in patients with AMI was lower in those with short reperfusion time even in the presence of high thrombus burden, which explain the possible correlation between thrombus burden degree and duration of reperfusion [20].

However, the no-reflow phenomenon can still occurs in patients with AMI and low thrombus burden and long reperfusion time. Even if the material potential to embolize is small, prolonged ischemia can disrupt the micro-vascular circulation and this disruption is known to be a key factor in the pathogenesis of no-reflow which is why an increased rate of no-reflow is seen in cases of prolonged reperfusion [21].

Then both groups were compared regarding the angiographic data with statistically significant higher thrombus burden degree $(56.3 \%$ vs $39.3 \%$ for no-reflow and reflow, respectively) and lower initial TIMI flow grade (81.2\% vs $63.1 \%$ for no-reflow and reflow, respectively) in the No-reflow group.

These data were exactly the same as Mazhar and Chen [14, 15] and close to Kirma and Zhou [1,5] who demonstrated that in addition to lower initial TIMI flow grade and higher thrombus burden degree, significantly more prevalence of total occlusion in the no-reflow patients but discordant to Tanaka who demonstrated that no statistically significant difference between reflow and no-reflow patients regarding all angiographic data [12] and Morishima who demonstrated that anterior infarction location was significantly higher in the no-reflow patients [13].

Thrombus burden was estimated using the thrombus scoring system [6] which was also used by $[1,5,15]$. 
A high thrombus burden commonly occurs in the setting of an occluded infarct artery and had been found to increase the risk of no-reflow as distal embolization of thrombotic debris can decrease myocardial perfusion and increase infarct size [22].

Pre-PCI good TIMI flow grade was strongly related to post-procedural TIMI 3 flow as patency of the IRA before PCI suggests a lower thrombus burden, resolution of vasospasm, spontaneous endogenous lysis of the thrombus and smaller infarct size [23].

Finally both groups were compared regarding the procedural data with statistically significant larger reference luminal diameter $(3.5 \pm 0.3$ vs. $3.2 \pm 0.3 \mathrm{~mm}$ for no-reflow and reflow, respectively), and longer lesion length (25.6 \pm 4.6 vs. $22.5 \pm 5.8 \mathrm{~mm}$ for no-reflow and reflow, respectively) in the No-reflow group.

These data were close to Kirma and Zhou who demonstrated that in addition to significant larger reference diameter and longer lesion length in the no-reflow group, the incidence of no-reflow was significantly lower in the patients treated by direct stenting than patients treated by stenting after balloon dilatation [1,5] but discordant to Chen who demonstrated that target lesion length and reference luminal diameter were not statistically significant [15] and Mazhar who demonstrated that downstream use of platelet GP IIb/IIIa inhibitors was significantly higher in the no-reflow patients [14].

Large vessels are able to contain large amounts of plaques containing lipid or thrombus and long lesions leads to slowing of the flow velocity. This would explain the high risk for noreflow observed in these patients after primary PCI [24].

\section{Limitations}

Single angiographic parameter was used to define noreflow. The microcirculation was not directly evaluated, for example by contrast echocardiography.

\section{Conclusions}

In the present study, no-reflow occurred in $16 \%$ of STEMI patients undergoing primary PCI and was more likely to be related to delayed reperfusion, low initial TIMI flow grade, high thrombus burden degree, large reference luminal diameter and long lesion length.

\section{Recommendations}

As there is no effective treatment for no-reflow once it has occurred, it is important to try, predict and prevent no-reflow from occurring especially among patients with one or more risk factors of no-reflow development.

Every attempt should be done to decrease reperfusion time as it's the most possible preventable predictor for no-reflow.

Education the public about heart attack symptoms and early presentation to hospital is very important.

Efforts to minimize delay due to diagnosis, transfer and intervention in STEMI patients is beneficial in reduction of no-reflow risk.

Interventional strategies such as deferred stenting, direct stenting, aspiration thrombectomy and longer antithrombotic therapy should be tested in a large controlled randomized trials and still to be considered in patients who are at higher risk of no-reflow such as patients with delayed presentation, low initial TIMI flow grade, high thrombus burden degree, large reference luminal diameter and those with long lesion length.

\section{References}

[1] Kirma C, Akin Izgi, Cihan Dundar, et al.: Clinical and Procedural Predictors of No-Reflow Phenomenon after Primary Percutaneous Coronary Interventions. Circ J 2008; 72: 716-721.

[2] Nagao K, Hayashi N, Kanmatsuse K, et al.: An early and complete reperfusion strategy for acute myocardial infarction using fibrinolysis and subsequent transluminal therapy: The FAST trial. Circ J 2002; 66: 576-582.

[3] Anderson JL, Karagounis L, Becker LC, et al.: TIMI perfusion grade 3 but not grade 2 results in improved outcome after thrombolysis for myocardial infarction: Ventriculographic, enzymatic, and electrocardiographic evidence from the TEAM-3 study. Circulation 1993; 87: 1829-1839.

[4] Cura F, L'Allier P, Kapadia SR, et al.: Predictors and prognosis of suboptimal coronary blood flow after primary coronary angioplasty in patients with acute myocardial infarction. Am J Cardiol 2001; 88: 124-128.

[5] Zhou H, Xiao-yan He, Shao-wei Zhuang, et al.: Clinical and procedural predictors of no-reflow in patients with acute myocardial infarction after primary percutaneous coronary intervention. Department of Cardiology, East Hospital, Tongji University School of Medicine, Shanghai 200120, China. World J Emerg Med, Vol 5, No 2, 2014.

[6] Gibson, CM, de Lemos, JA, et al.: "Combination therapy with abciximab reduces angiographically evident thrombus in acute myocardial infarction: a TIMI 14 substudy. Circulation. 2001, 103 (21): 2550-4.

[7] Bonaa KH, Mannsverk J, Wiseth R, et al.: Drug-eluting or bare-metal stents for coronary artery disease. N Engl J Med 2016; 375(13):1242-1252.

[8] Elgendy IY, Mahmoud AN, Kumbhani DJ, et al.: Complete or culprit only revascularization for patients with multi-vessel coronary artery disease undergoing percutaneous coronary intervention: a pair wise and network meta-analysis of randomized trials. JACC Cardiovasc Interv 2017; 10 (4):315-324.

[9] Ten Berg JM, van't Hof AW, Dill T, et al.: Effect of early, prehospital initiation of high bolus dose tirofiban in patients with ST-segment elevation myocardial infarction on short- and long-term clinical outcome. J Am Coll Cardiol 2010; 55 (22):2446-2455.

[10] Kelbaek H, Hofsten DE, Kober L, et al.: Deferred versus conventional stent implantation in patients with ST-segment elevation myocardial infarction (DANAMI 3-DEFER): an open-label, randomized controlled trial. Lancet 2016; 387(10034):2199-2206. 
[11] Watanabe T, Nanto S, Uematsu M, et al.: Prediction of NoReflow Phenomenon after Successful Percutaneous Coronary Intervention in Patients with Acute Myocardial Infarction Circulation Journal Vol.67, August 2003.

[12] Tanaka A, Kawarabayashi T, Yoshiharu et al.: No-Reflow Phenomenon and Lesion Morphology in Patients with Acute Myocardial Infarction Circulation. 2002; 105:2148-2152.

[13] Morishima I, Sone T, Okumura K, et al.: Angiographic noreflow phenomenon as a predictor of adverse long-term outcome in patients treated with percutaneous transluminal coronary angioplasty for first myocardial infarction. J Am Coll Cardiol. 2000; 36:1202-1209.

[14] Mazhar J, Maschicharan M, Farshid A. Predictors and outcome of no reflow post primary percutaneous coronary intervention for ST-elevation myocardial infarction. IJC Heart Vasc. 2016; 10:8-12.

[15] Chen Y, Wang C, Xinchun, et al.: Independent no-reflow predictors in female patients with ST-elevation acute myocardial infarction treated with primary percutaneous coronary intervention Heart Vessels (2012) 27:243-249.

[16] Niccoli G, Burzotta F, Galiuto L, et al.: Myocardial no-reflow in humans. J Am Coll Cardiol. 2009; 54:281-292.

[17] Reffelmann $\mathrm{T}$ and Kloner RA. The no-reflow phenomenon: Basic science and clinical correlates. Heart 2002; 87: 162168

[18] B. G. Schwartz and R. A. Kloner, Coronary no reflow, J. Mol. Cell. Cardiol. 52 (4) (2012) 873-882.
[19] Nagata Y, Usuda K, Uchiyama A, et al.: Characteristics of the pathological images of coronary artery thrombi according to the infarct-related coronary artery in acute myocardial infarction. Circ J 2004; 68: 308-314.

[20] Yip HK, Chen MC, Chang HW, et al.: Angiographic morphologic features of infarct-related arteries and timely reperfusion in acute myocardial infarction: predictors of slowflow and no-reflow phenomenon. Chest 2002; 122:1322-32.

[21] De Luca G, Suryapranata H, Zijlstra F, et al.: Symptom-onsetto-balloon time and mortality in patients with acute myocardial infarction treated by primary angioplasty. J Am Coll Cardiol 2003; 42: 991-997.

[22] Dong-bao, H. Qi, L. Zhi, et al.: Predictors and long-term prognosis of angiographic slow/no-reflow phenomenon during emergency percutaneous coronary intervention for STelevated acute myocardial infarction, Clin. Cardiol. 33 (12) (2010) E7-12.

[23] De Luca G, Ernst N, Zijlstra F, et al.: Preprocedural TIMI flow and mortality in patients with acute myocardial infarction treated by primary angioplasty. J Am Coll Cardiol 2004; 43: $1363-1367$

[24] Katayama T, Kubo N, Takagi Y, et al.: Relation of atherothrombosis burden and volume detected by intravascular ultrasound to angiographic no-reflow phenomenon during stent implantation in patients with acute myocardial infarction. Am J Cardiol 2006; 97: 301-304. 